\title{
THE POLYMERIZATION OF PROTEINS
}

\section{Adenosine Triphosphate and the Polymerization of Actin}

\author{
BY KOLOMAN LAKI,* WILLIAM J. BOWEN, AND ARIEL CLARK
}

(From the Laboratory of Physical Biology, Experimental Biology, and Medicine Institute, National Instilutes of Health, Bethesda)

(Received for publication, November 9, 1949)

Banga and Szent-Györgyi (1) have found that two kinds of myosin can be obtained from rabbit muscle depending on the time of extraction of the muscle. Muscle extracted for 24 hours yields myosin which is very viscous (myosin B) and the viscosity drops on the addition of adenosine triphosphate (ATP), while muscle extracted for 20 minutes yields myosin which is less viscous and the viscosity does not change on addition of ATP (myosin A). Straub (2), studying the properties of the two forms of myosin, obtained a highly viscous protein extract from acetone-dried rabbit muscle which, when added to myosin $\mathrm{A}$, changed its properties to myosin $\mathrm{B}$. In this reaction this viscous protein, which was called actin, combined with myosin to form a complex (actomyosin), the most important property of which is contractility (2). Straub's most interesting observation was that this viscous protein can be obtained, under specific conditions, in a non-viscous form, and that this protein solution becomes very viscous upon the addition of salts (3). He considered this change to be a polymerization of the globular actin to a fibrous form (3). Electron microscope investigations of Jakus and Hall (4) actually demonstrated the formation of long continuous fibers. Rozsa, Szent-Györgyi, and Wyckoff (5) have shown with the aid of the electron microscope that the change of globular actin into the fibrous form takes place in two steps. First, the small globules (molecular weight $\sim 70,000$ ) unite into large globular units (molecular weight $\sim 1,500,000)$. Then, these units unite to form threads. Thus actin, besides being a very important contractile constituent of muscle fiber, constitutes an interesting example of protein polymerization.

In this paper experiments are described which show that adenosine triphosphate is involved in the polymerization of actin. It has recently come to our attention that Straub $(6)^{1}$ also found that ATP is instrumental in bringing about actin polymerization.

* Special Research Fellow, National Institutes of Health.

${ }^{1}$ After the manuscript of this paper was submitted, Straub published an abstract for the First International Congress of Biochemistry in which he states that adenosine triphosphate must be regarded as the prosthetic group of actin, the removal of which renders actin non-polymerizable. 


\section{EXPERTMENTAI}

Actin for these experiments was prepared according to Feuer et al. (7). Solutions of actin were prepared by extracting $1 \mathrm{gm}$. of dried muscle residue with $20 \mathrm{ml} . \mathrm{CO}_{2}$ free water for 30 minutes and filtering through a Buchner funnel. Such solutions contain actin in the globular, or non-polymerized form, which has been called G-actin (3).

Spectrophotometric Studies.-Feuer et al. (7) show an absorption spectrum of actin which has a maximum at about $260 \mathrm{~m} \mu$. Such a maximum resembles that of a nucleoprotein more than that of a protein, and it may be due to nucleotides bound to the protein. We observed the same spectrum of actin and extended observations to ascertain what substances present cause this alteration of the spectrum of a protein, and whether their removal has any influence on the polymerizability of actin.

Absorption spectra of actin and its components were obtained as follows: A solution of actin containing $4.1 \mathrm{mg}$. dry material per $\mathrm{ml}$. was diluted 5 times to obtain its absorption spectrum. The protein of another solution was precipitated isoelectrically by the addition of $\mathrm{M}$ acetic acid until $\mathrm{pH} 4.7$ was obtained. The suspension was then centrifuged at $1000 \mathrm{~g}$ for 5 minutes. The precipitated protein was redissolved in water by the addition of a few drops of ammonia $(\mathrm{pH} \mathrm{7-8)}$. This clear solution contained $3.1 \mathrm{mg}$. dry material per $\mathrm{ml}$. Its absorption spectrum was made after diluting 5 times. The absorption spectrum of the supernatant of the above precipitation was made after diluting twice. The analyses were made with a Beckman quartz spectrophotometer DU Model.

The maximum absorption of the unaltered actin was found to be at $260 \mathrm{~m} \mu$ (Fig. 1), about $17 \mathrm{~m} \mu$ below the maximum of a protein. After acid precipitation and redissolving in alkali the maximum absorption of the actin was at 275 $\mathrm{m} \mu$, which is near the maximum of myosin (Fig. 1) and other proteins. The maximum absorption of the supernatant fluid from isoelectric precipitation was obtained at $258 \mathrm{~m} \mu$, and another maximum between 252 and $253 \mathrm{~m} \mu$ was indicated. The supernatants of some actin preparations had only one absorption maximum at $250 \mathrm{~m} \mu$.

These findings indicate that actin contains some substance other than protein. The spectra of the supernatants suggest the presence of adenine or hypoxanthine. Other data not presented here indicate that adenine and hypoxanthine are present in proportions differing from preparation to preparation.

Like Feuer et al. (7) we also found that after isoelectric precipitation actin loses its polymerizability. The addition of the supernatant to the isoelectric precipitate restores the polymerizability, showing that some factors in the supernatant are necessary for the polymerization. This fact and the spectroscopic findings suggested studying whether hypoxanthine or adenine nucleotides participate in the polymerization of actin. As a start we estimated the ATP present in actin preparations.

Estimation of ATP.-The protein of the G-actin solution was removed by filtration, after acid and heat precipitation, and $0.5 \mathrm{ml}$. of the filtrate, after neutralization, 


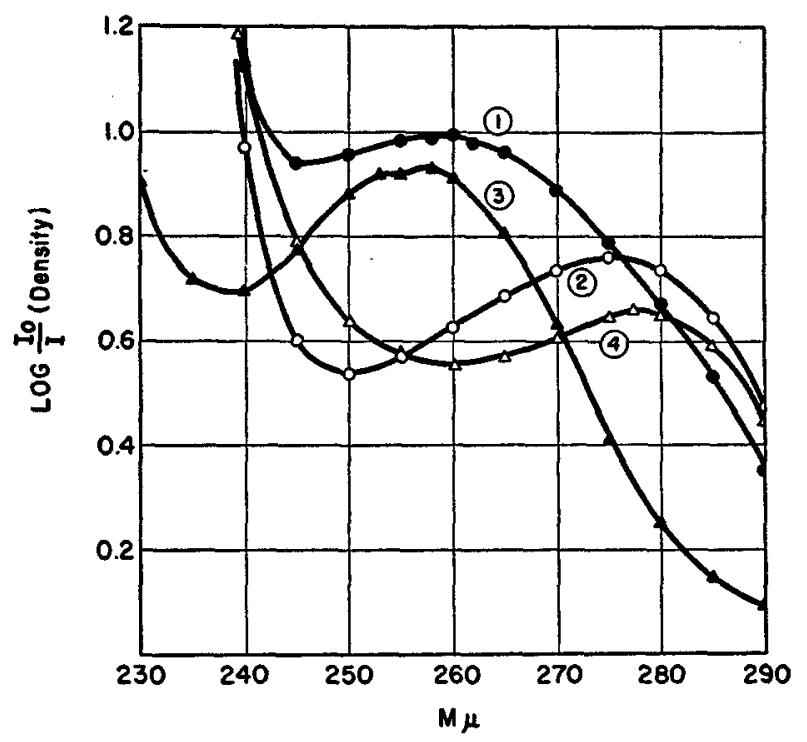

Fig. 1. Absorption curves. Curve 1, actin- $-0.82 \mathrm{mg}$. protein per ml. Curve 2, actin precipitated isoelectrically, neutralized, and redissolved in water $-0.66 \mathrm{mg}$. protein per ml. Curve 3, supernatant of actin precipitation. Curve 4 , myosin $-0.54 \mathrm{mg}$. protein per $\mathrm{ml}$.

TABLE I

ATP Content of Actin

The test used depends upon the reduction of triphosphopyridine nucleotide (TPN) by hexose-6-phosphate which is formed by transphosphorylation from ATP. The amounts of ATP found are based upon the amount of TPN reduced.

\begin{tabular}{c|c|c|c}
\hline \multirow{2}{*}{ Preparation } & Dry weight & \multicolumn{2}{|c}{ ATP } \\
\cline { 2 - 3 } & mg. per ml. & Globular & Globular redissolved \\
\hline & 4.1 & $\mu$ M per ml. & $\mu$ M per ml. \\
1 & 4.6 & 0.020 & 0.005 \\
2 & 0.010 & 0.004 \\
\hline & & Globular & Fibrous \\
\hline 3 & 9.3 & 0.100 & 0.090 \\
4 & 6.6 & 0.062 & 0.050 \\
5 & 4.1 & 0.034 & 0.030 \\
6 & - & 0.020 & 0.016 \\
\hline
\end{tabular}

was tested for ATP. The protein of another portion of the same solution was precipitated isoelectrically at $\mathrm{pH} 4.7$ and redissolved in water by the addition of ammonia. This solution was then treated as the G-actin above and its filtrate tested for ATP. This experiment was done twice (actins 1 and 2, Table I). 
The ATP content of filtrate from polymerized actin was also determined in the same manner. Extracts of each of 4 actin preparations were divided in to 2 portions. One portion was polymerized by making the extract $0.1 \mathrm{M} \mathrm{KCl}$. Then the protein in each portion was removed as above and $0.5 \mathrm{ml}$. of the filtrate tested for ATP (actins 3 to 6 , Table I).

The test to estimate ATP is enzymatic and depends upon the ultimate use of the terminal phosphate of ATP to reduce triphosphopyridine nucleotide (TPN). ${ }^{2}$ The reaction mixture contained solution to be estimated for its ATP content, hexokinase, glucose, zwischenferment, ${ }^{3}$ and TPN. The reduction of TPN was followed spectrophotometrically at $340 \mathrm{~m} \mu$. The test is specific for ATP.

The results of these tests (Table I) show that the ATP content of G-actin varies from preparation to preparation. This variation is roughly proportional to the dry weight of the tested actin solutions. The fact that ATP is separated from actin when it is isoelectrically precipitated shows that the ATP in the original extract is either in solution or very loosely bound to the protein molecules. The ATP in the precipitated and redissolved (in water) actin is not in excess of the amount that could be carried into it by fluid in the precipitate.

The ATP contents of the supernatants of the polymerized actins were smaller than those of globular actin. These differences were small but consistent and suggest that ATP is utilized in the process of polymerization.

In spite of these findings we were not able to restore the polymerizability of precipitated actin by exchanging the supernatant for an ATP solution. This indicates that some factor other than ATP is also involved in polymerization. It can be seen by comparing the amount of ATP found in the analyses $(0.034 \mu \mathrm{M})$ above with the amount of adenine calculated from the spectrum $(0.11 \mu \mathrm{M})$ at its maximum absorption $(260 \mathrm{m \mu})$ that the ATP present is only a fraction of the total adenine. Nevertheless, the experiment described in the next section shows that ATP is somehow involved in the polymerization of actin.

Factors of Polymerization.-The polymerization of actin can be brought about in two ways: either by adding salts $(3,5,7)$ or acid $(\mathrm{pH} 6)$ to the G-actin solutions. Such solutions of polymerized actin are referred to as $\mathbf{F}$-actin. To avoid acid-induced polymerization all the following experiments were done at $\mathrm{pH}$ 8.3. The experiments above led us to test the influence of ATP upon this polymerization by removing the ATP naturally present in G-actin. Removal was accomplished by incubation of G-actin with hexokinase (in presence of glucose) or creatine.

${ }^{2}$ We are indebted to Dr. Arthur Kornberg for the procedure of this test prior to its publication and for some of the materials used in it. We are also indebted to Professor C. F. Cori for a sample of triphosphopyridine nucleotide.

'Zwischenferment was prepared according to Warburg, O., and Christian, W., Biochem. Z., 1932, 254, 438. 
The experiments were done in the following manner: The process of polymerization was followed at $25^{\circ}$ by measuring the time of efflux in a Cannon-Fenske viscometer until no further changes occurred. The times of efflux were converted to apparent specific viscosities ( $\eta \mathrm{sp}$ ) by substituting in the following equation:

$$
\eta \mathrm{sp}=\frac{t}{t_{0}}-1
$$

where $t=$ seconds of efflux of a solution containing actin, buffer, and salt and $t_{9}=$ seconds of efflux of a solution containing only buffer and salt.

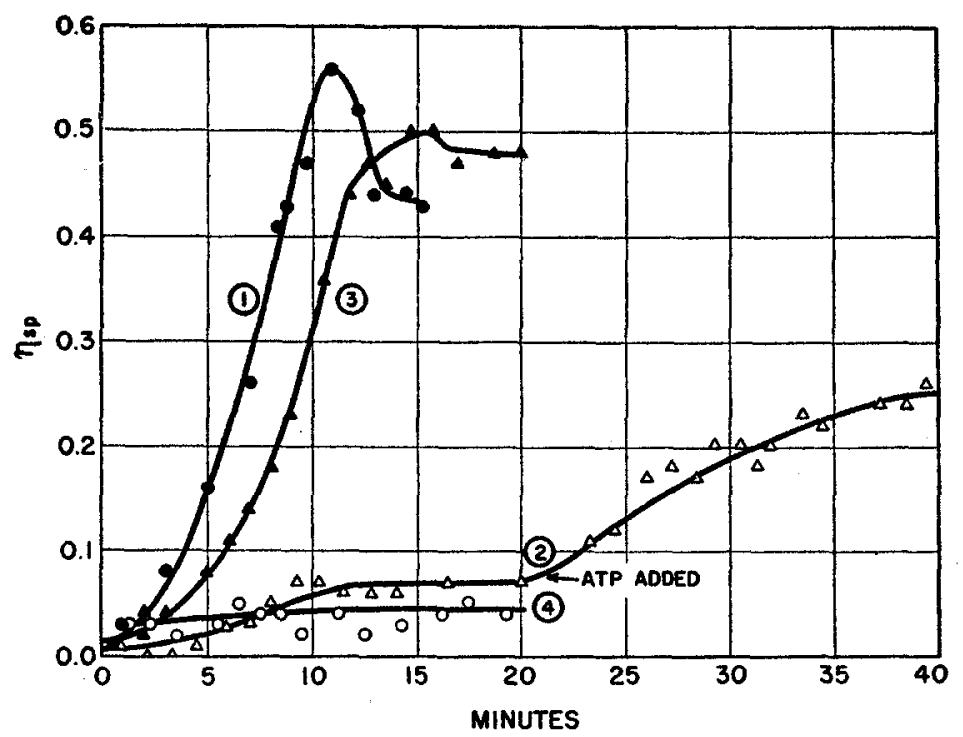

Fig. 2. Effects of hexokinase and adenosine triphosphate (ATP) on the polymerization of actin. Curve 1, untreated actin. Curve 2, actin incubated with hexokinase. The second part of the curve (beyond 21 minutes) shows the partial restoration obtained by the addition of ATP. Curve 3, actin incubated with hexokinase and ATP. Curve 4, actin incubated with hexokinase, ATP, and excess glucose.

Four ml. of actin solution in a viscometer were incubated with the following materials in separate experiments: (a) $0.1 \mathrm{ml}$. hexokinase; ${ }^{4}(b) 0.1 \mathrm{ml}$. hexokinase and 0.625 micromole of ATP; ${ }^{5}(c) 0.1 \mathrm{ml}$. hexokinase, 0.625 micromole ATP and glucose, in

4 The hexokinase was generously supplied by Dr. Arthur Kornberg. The purity of the preparation was about 10 per cent and contained 2500 units per ml.

${ }^{5}$ Two preparations of ATP were used. One was prepared according to SzentGyörgyi (8) from the calcium salt and contained $5.3 \mu \mathrm{M}$ of inorganic $\mathrm{P}$ and $6.25 \mu \mathrm{M}$ ATP per ml. (27 per cent of the total adenine present). The other was prepared from the dibarium salt (Nutritional Biochemicals Corporation) and contained $0.27 \mu \mathrm{M}$ inorganic P and $3.53 \mu \mathrm{M}$ ATP per ml. (nearly 100 per cent of the adenine content). 
stoichiometric excess of that needed to dephosphorylate the ATP; and (d) water sufficient to equalize dilution of above additions (controls). After 20 minutes' incubation $2.0 \mathrm{ml}$. veronal-acetate buffer, $\mathrm{pH} 8.3$, and $1.0 \mathrm{ml}$. of a salt mixture $(0.8 \mathrm{M} \mathrm{KCl}$ and $0.008 \mathrm{M} \mathrm{MgCl}_{2}$ ) were added to each viscometer. Then polymerization was followed simultaneously in each viscometer by ascertaining the viscosities alternately every 2 or 3 minutes.

Fig. 2 (curve 2) shows that incubation of G-actin with hexokinase inhibits polymerization and that the addition of ATP partially restores polymerizability. Curve 3 shows the efficacy of ATP in counteracting the inhibition of polymeri-

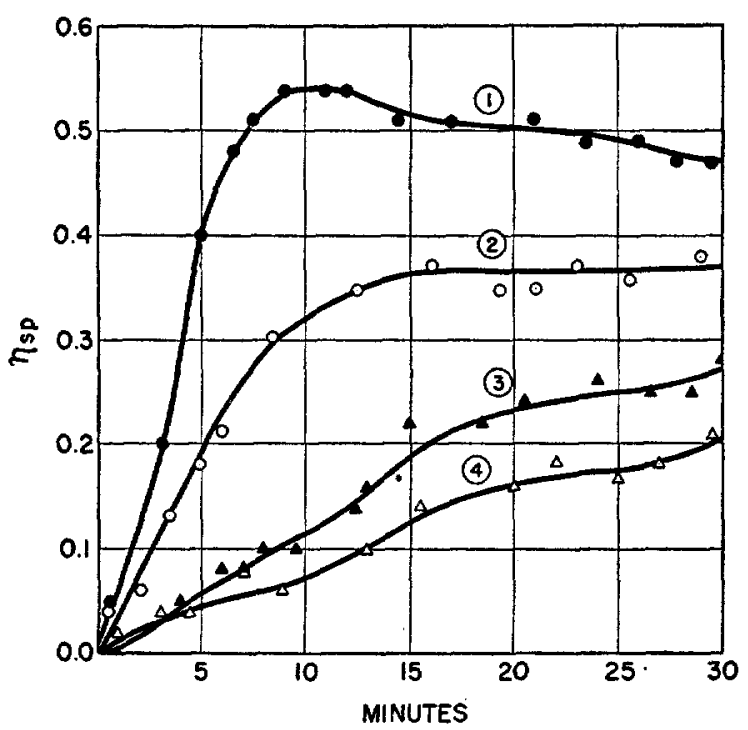

FIG. 3. Effects of creatine on the polymerization of actin. Curve 1, untreated actin. Curve 2, $2 \mathrm{mg}$. creatine. Curve $3,4 \mathrm{mg}$. creatine. Curve 4, $6 \mathrm{mg}$. creatine.

zation of actin by hexokinase. Curve 4 shows that actin in the presence of hexokinase, ATP, and excess glucose does not polymerize.

It was found that our actin preparations contain the enzyme, ATP-creatine phosphopherase. Therefore, similar experiments were performed in which G-actin was incubated with creatine instead of with hexokinase and glucose. The results of these experiments (Fig. 3) show that creatine also inhibits polymerization. A direct relationship between the degree of inhibition of actin polymerization and the amount of creatine present was shown by the results of these experiments (Fig. 3) by incubating G-actin with amounts of creatine varying from 2 to $6 \mathrm{mg}$. 
SUMMARY

It was found that actin solutions contain ATP. Experiments in which hexokinase and creatine were added to actin show that the ensuing dephosphorylation of ATP affects actin so that it will not polymerize.

\section{BIBLIOGRAPHY}

1. Banga, I., and Szent-Györgyi, A., Studies Inst. Med. Chem. Univ. Szeged, 1941-42, 1,5 .

2. Straub, F. B., Studies Inst. Med. Chem. Univ. Szeged, 1942, 2, 3.

3. Straub, F. B., Studies Inst. Med. Chem. Univ. Szeged, 1943, 3, 23.

4. Jakus, M. A., and Hall, C. E., J. Biol. Chem., 1947, 167, 705.

5. Rozsa, G., Szent-Györgyi, A., and Wyckoff, R. W. G, Biochim. et Biophys. Acta, 1949, 3, 561.

6. Straub, F. B., personal communication.

7. Feuer, G., Molnar, F., Pettko, E., and Straub, F. B., Hung. Acta Physiol., 1948, $1,150$.

8. Szent-Györgyi, A., Muscular Contraction, New York, Academic Press, Inc., 1947, 133. 